\title{
Designing Zirconium Coated Polystyrene Colloids and Application
}

\author{
Diana Chira and Seong S. Seo \\ Department of Natural Sciences, Albany State University, 504 College Drive, Albany, GA 31705, USA \\ Correspondence should be addressed to Seong S. Seo, seong.seo@asurams.edu
}

Received 15 December 2008; Revised 20 March 2009; Accepted 29 March 2009

Recommended by Yongxiang Li

\begin{abstract}
A simple technique has been developed to prepare core colloids that are modified using zirconium oxychloride, based on heating a solution of core colloid composites, consisting of poly (ethylenimine) (PEI) and zirconium oxychloride. The interaction of zirconium oxychloride with the polystyrene (PS) core colloids has been investigated using Fourier transform-infrared spectroscopy (FT-IR), energy dispersive X-ray spectroscopy (EDX), and scanning electron microscopy (SEM) data. FT-IR studies confirm the occurrence of amine groups present in PEI which are oxidized to carboxyl groups after the reaction. The EDX data and the SEM images confirm the presence of zirconium particles immobilized on the polystyrene surfaces. Demeton, a highly toxic nerve agent, was used due to its ability to easily bind through its organophosphate group illustrating a practical application of the PS-PEI$\mathrm{Zr}$ particles. Attenuated Total Reflection (ATR) Spectroscopy was used to assess the interactions between the toxic nerve agent demeton-S and the PS-PEI-Zr particles. The results show that the presented technique for coating polystyrene core colloids with zirconium was successfully accomplished, and the newly formed particles easily bond with demeton agents through the $\mathrm{P}=\mathrm{O}$ functional group.
\end{abstract}

Copyright ( $) 2009$ D. Chira and S. S. Seo. This is an open access article distributed under the Creative Commons Attribution License, which permits unrestricted use, distribution, and reproduction in any medium, provided the original work is properly cited.

\section{Introduction}

Recently, numerous synthetic mechanisms have been employed for surface adsorption of metal nanoparticles (NPs) onto colloidal inorganic and organic spheres [1-6]. A particularly successful method of depositing the metal NPs on the surface of the colloids involves the use of an intermediate linker poly (ethylenimine), PEI, which can bind both transition metal ions, such as zirconium, and negatively charged colloids [7-10]. Additionally, the PEI serves as the reducing agent in the conversion of the metal ions to the metal NPs [11]. In such a scheme, the core component, usually polystyrene (PS) or silicon dioxide $\left(\mathrm{SiO}_{2}\right)$, serves as a supporting structure, and the outer NPs predominantly exhibit the properties that are attributed to the metal NP-core colloid composites. The advantages derived from these hybrid materials can be seen in their remarkable attributes which include enhanced conductivity, temperature stability, optical, and catalytic activity [12-14].
Over the years, there has been considerable interest surrounding the fabrication of core-shell spheres that consist of templated cores coated with shells that have different chemical compositions. These core-shell spheres often exhibit properties that are substantially different from those of the templated core (increased stability, higher surface area, different magnetic, and optical properties), making them attractive from both a scientific and technological standpoint [15-17]. Furthermore, the properties of core-shell particles can also be tailored in a controlled fashion by independently altering the composition, dimension, and structure of the core or shell. Application for such core-shell spheres is diverse, including biosensors [18], chemical sensors [19], and so forth.

Organophosphates (OPs) are well-known neurotoxins; they disrupt the cholinesterase enzyme that regulates acetylcholine [20-22], a neurotransmitter needed for proper nervous system function. Because of their high neurotoxicity, OP compounds have been exploited for use as pesticides and as nerve agents with application as chemical and biological 


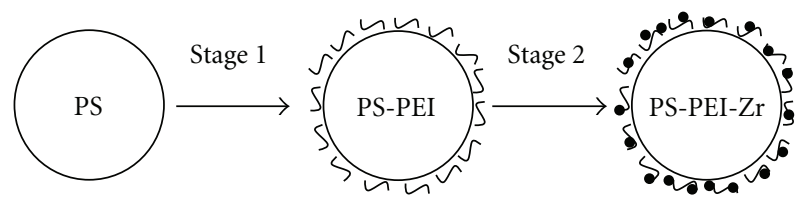

Scheme 1: Procedure for coating PS colloids with metal.

warfare agents. As a result of the high toxicity of OPs, fast and effective detection of these toxic agents in the environment, public places, or workplaces and the monitoring of individual exposures to chemical warfare agents have become increasingly important for national security and health protection purposes [23-25]. Early detection and detection of low concentrations of OPs still remain an extremely difficult challenge. Such information may give an indication of terrorist activity, allowing proper procedures to be followed to mitigate potential danger. Analysis of OPs in environmental and biological samples is routinely carried out using analytical techniques, such as gas or liquid chromatography and mass spectrometry [26].

For OP detection, enzyme-biosensors of inhibition and noninhibition systems are mostly based on the immobilization of acetylcholinester (AChE) [27], tyrosinase enzyme (Tyr) [28], alkaline phosphatase (ALP) [29], or organophosphorus hydrolase (OHP) $[30,31]$ onto various electrodes. Due to their high sensitivity and selectivity toward OPs, these have been proposed for field screening of OP neurotoxins [32]. However, while these specific antibodies against OP pesticides have been recently developed for enzymelinked immunoassay and immunosensors [33, 34], acetylcholinesterase is commercially available but OP hydrolase and antibodies against OPs are still only produced in laboratories, which limits wide applications of biosensors. Demeton is also used as pesticide, which on most metal oxides, in our case zirconium and adsorbs through the $\mathrm{P}=\mathrm{O}$ functional group. IR spectroscopy in combination with Attenuated Total Reflection (ATR) spectroscopy is one of the most widely used techniques for surface infrared analysis. ATR-IR has been used for studying processes at surfaces or in films, such as adsorption [35-37] and diffusion [38-40].

The aim of this paper is to treat polystyrene colloids with zirconium oxychloride in order to create a hybrid material which may find utility as an organophosphate sensor. The composite colloids are obtained by coating PS with PEI followed by the addition of $\mathrm{ZrO}_{2}$ (Figure 1). In this reaction scheme, PEI is relatively inert and thus does not have a significant effect, if any, on the ultimate formation of the NPs. The advantages of this method are simple operation, easy large scale production, and the generality for various core colloids. The zirconium acts as a selective probe targeting the phosphate group of the demeton which upon bonding shows a change in the ATR spectrum. Other inorganic ions have also found to have an affinity for $\mathrm{P}=\mathrm{O}$ functional groups, such as $\mathrm{Mg}$, thus paving the way for future research into different colloidal compositions for OP detection.
In this paper, we report a novel method for detecting OP chemical nerve agents by incorporating the enhanced properties of zirconium coated polystyrene core colloid composites with the strong affinity for chemical nerve agents. Polystyrene was utilized for the core component with the outer layer being comprised of zirconium NPs. We characterized the PS-PEI-Zr colloids by using SEM, EDX, and FT-IR spectroscopy. The prepared zirconium colloids were placed on the ATR ZnSe crystal forming a thin layer used for the detection of demeton-S.

\section{Experimental Section}

2.1. Materials. Poly (ethylenimine) (PEI), polystyrene (PS) colloids with an average size of $210 \mathrm{~nm}$, sodium phosphate buffer, zirconium oxychloride, sodium citrate $\left(\mathrm{C}_{6} \mathrm{H}_{5} \mathrm{O}_{7} \mathrm{Na}_{3} .2 \mathrm{H}_{2} \mathrm{O}\right)$, absolute ethanol, ammonium hydroxide solution $\left(\mathrm{NH}_{4} \mathrm{OH}, 25 \%\right)$, formaldehyde ( $\mathrm{HCHO}$, $37 \%$ ), and Demeton-S were all obtained from SigmaAldrich.

2.2. Modification of Core Colloids with PEI (PS-PEI). $1 \mathrm{~g}$ of PS colloids was dispersed in $50 \mathrm{~mL}$ of phosphate buffer solution with a pH of 6.5 (solution A). $0.1 \mathrm{~g}$ of PEI was then dissolved in $5 \mathrm{~mL}$ of phosphate buffer to produce solution B (mass ratio of PEI to PS was 1 : 10). Solution $B$ was stirred at room temperature $\left(25^{\circ} \mathrm{C}\right)$ for 1 hour followed by three cycles of centrifugation $(20000 \mathrm{rpm})$ and washing with $10 \mathrm{~mL}$ of water.

2.3. Preparation of PS-PEI-Zr. PEI modified PS colloid solution was dispersed in $100 \mathrm{~mL}$ water, and $0.15 \mathrm{~g}$ of zirconium oxychloride was added and then heated for one hour at $100^{\circ} \mathrm{C}$. After cooling to room temperature, the ammonia and sodium citrates were added into the dispersion solution. $12 \mathrm{~mL}$ of sodium citrate $(1 \mathrm{M})$ was added to result in a $1: 1$ molar ratio of zirconium oxychloride to sodium citrate. Dropwise addition of $\mathrm{NH}_{4} \mathrm{OH}(1 \mathrm{M})$ were used to bring the $\mathrm{pH}$ up to 10 . Then, the $\mathrm{HCHO}$ diluted with ethanol was added. After completion, the solid was isolated by 3 cycles of centrifugation/washing.

2.4. Characterization. FTIR and ATR data were recorded with a Perkin Elmer Spectrum BX FT-IR System. The sample for the FTIR measurement was prepared by grinding a dry PS-PEI-Zr sample with $\mathrm{KBr}$ into fine powders which were then pressed at 20000 psi into pellets. Scanning electron microscopy (SEM) was performed with a JSM-5900.

2.5. Demeton Adsorption on PS-PEI-Zr. $10 \mu \mathrm{l}$ of the prepared PS-PEI-Zr colloids were placed on a Miracle Universal Plate composed of ZnSe crystal (PIKE Technologies, Single Reflection Horizontal ATR) and allowed to dry, forming a thin layer. $10 \mu \mathrm{L}$ of $1 \mathrm{mM}$ demeton-S solution were then added to the dry PS-PEI-Zr thin layer, and the ATR spectrum was obtained at a $4 \mathrm{~cm}^{-1}$ resolution with a spectral range of $750-4000 \mathrm{~cm}^{-1}$. Following this, an additional $10 \mu \mathrm{L}$ of the 
demeton-S solution were added to give a cumulative $20 \mu \mathrm{L}$, for which the ATR spectrum was obtained in each case.

\section{Results and Discussion}

In the present study, PEI-modified polystyrene colloids were used as the templates for the deposition of zirconium nanoparticles. Tian et al. [41, 42] have previously demonstrated that PEI acts as a reductant in the conversion of transition compounds into metallic particles. Moreover, through the use of TEM, XPS, and XRD they were able to identify the metallic particles whether it was $\mathrm{Ag}, \mathrm{Au}$, or another transition, and the exact oxidation state was thus obtained. In this study we use zirconium oxychloride which is reduced to metallic zirconium particles upon being adsorbed onto the PEI modified colloids. This chemistry is critical in the formation of the metal nanoparticles; otherwise, the PEI will simply be coated with a layer of oxychloride, changing the chemical properties we seek.

Following synthesis, the prepared composites were characterized by FT-IR spectroscopy, scanning electron microscopy (SEM), and EDX. Figure 1 shows SEM images of the prepared composites at different magnifications, showing the deposition of zirconium onto the core polystyrene colloids. A larger central PS core can be observed, and on the superficial surface, small protrusions of the $\mathrm{Zr}$ metal are visually attached which are shown by a porous network on the surface of the colloids as opposed to bare PS which shows a smooth consistent surface. Scanning electron microscopy images were obtained on a JSM-5900 which was operated at $30 \mathrm{kV}$. The SEM analysis was consistent and reproducible with a representative area shown in Figure 2. The synthesized PS-PEI-Zr composites were suspended in water, dispersed then on glass, and coated with approximately $10 \mathrm{~nm}$ of AuPd. The lighter edges is an edge effect, that is, secondary electrons can be collected from the top and bottom surfaces of the sphere edges, leading to an enhanced secondary electron signal. Figure 2 displays an EDX spectrum which quantitatively detects the constituent elements. The spectrum showed Zr LA1, Zr LB1, Zr LB3, Zr L1, Zr KA1, and $\mathrm{Zr} \mathrm{KB1}$ peaks, indicating the presence of the third layer of zirconium oxychloride on the polystyrene colloids. Other peaks detected in the EDX spectrum included $\mathrm{Cl} \mathrm{KA1,} \mathrm{Cl}$ $\mathrm{KB} 1$, and $\mathrm{O} \mathrm{KA} 1$, exposing elements such as chlorine and oxygen, a result of the deposition of zirconium oxychloride on the polystyrene colloids.

In Figure 3, the IR spectra of PS, PS-PEI, and PS-PEI-Zr composites detect a peak at $2916 \mathrm{~cm}^{-1}$ which corresponds to the stretching vibration of $\mathrm{C}-\mathrm{H}$, also indicating the presence of PEI on the PS colloids. At around $1700 \mathrm{~cm}^{-1}$, the presence of the $\mathrm{C}=\mathrm{O}$ stretching of carboxyl is detected. Analyzing the PS-PEI curve, it is noticeable that the $\mathrm{C}=\mathrm{O}$ bond is not present anymore due to the consummation of the carboxyl group of PS with PEI and $\mathrm{Zr}$ nanoparticles in the colloidal modifications. There are two more prominent peaks present at 1492 and $1452 \mathrm{~cm}^{-1}$ which are a result of the antisymmetric and symmetric stretching of $\mathrm{O}-\mathrm{C}-\mathrm{O}$. These two are easily detectable in the PS curve, however,

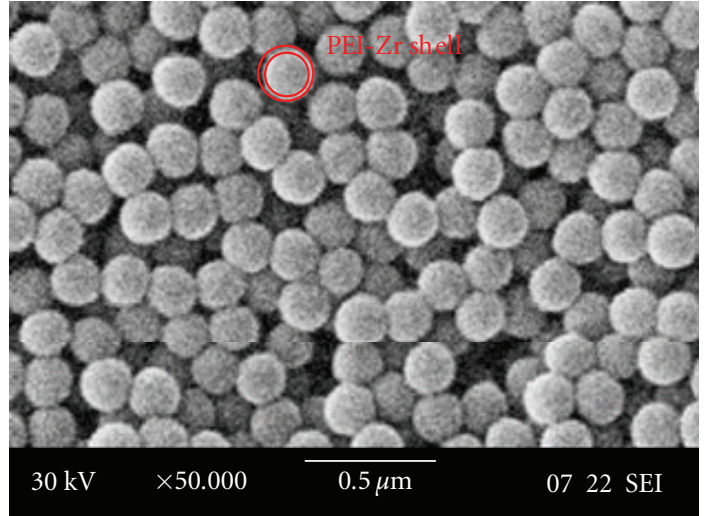

Figure 1: SEM image of PS-PEI-Zr spheres.

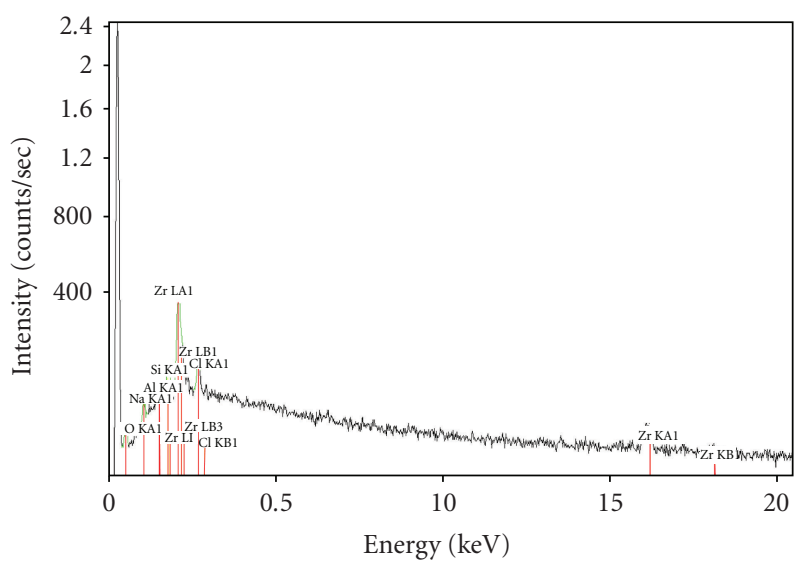

FIGURE 2: EDX of PS-PEI-Zr.

it diminishes in the PS-PEI curve and disappears almost completely in the PS-PEI-Zr curve. This is again due to the expenditure of the functional group in the formation of PSPEI and PS-PEI-Zr colloids. The peak around $3500 \mathrm{~cm}^{-1}$ is characteristic of the $\mathrm{N}-\mathrm{H}$ stretching vibration from the PEI, thus it is not present in the PS curve and does not considerably reduce in the PS-PEI-Zr curve. Analysis of the reaction mechanism occurring between PEI and $\mathrm{Zr}$ has previously been studied and reported by IR spectroscopy [43]. It was shown that two new IR bands involving interactions between $\mathrm{Zr}$ (IV) (Lewis Acid) and the nitrogen of the amine group (Lewis base) in PEI were present in the IR spectrum. A broad band in the $1580-1560 \mathrm{~cm}^{-1}$ region is observed both for aromatic amines (polyvinyl imidazole) and for aliphatic amines (polyethyleneimine), which can be easily assigned to the $\mathrm{NH}_{2}$ asymmetric bending mode for the latter. A second band is observed in the $1450-1350 \mathrm{~cm}^{-1}$ range, depending on the nature of the amine group.

Figure 4 shows the ATR spectra for consecutive additions of $10 \mu \mathrm{L}$ aliquots of a $0.06 \mathrm{M}$ demeton solution to a thin layer of the PS-PEI-Zr colloids. Analysis of the ATR data and the relevant peaks shows that the binding of the PSPEI-Zr colloids to demeton has been successfully achieved. All four peaks at $2350 \mathrm{~cm}^{-1}$ are designated peaks for the 




FIGURE 3: FT-IR spectra of PS, PS-PEI, and PS-PEI-Zr.

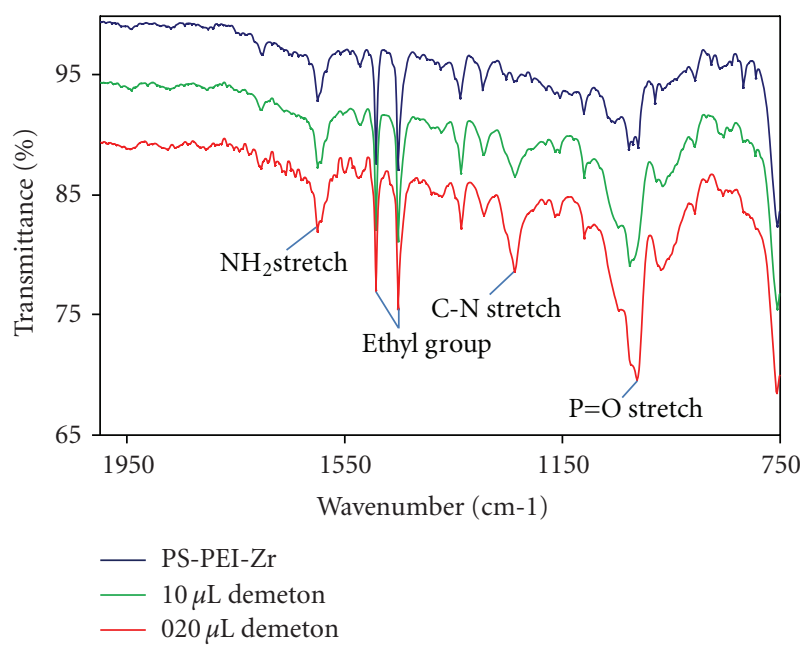

Figure 4: ATR spectrum PS-PEI-Zr with 10 and $20 \mu \mathrm{L}$ of $0.06 \mathrm{M}$ demeton.

presence of zirconium. Zirconium is not only present in the Ps-PEI-Zr compound form, but also in its binding to demeton. At $1010 \mathrm{~cm}^{-1}$ there is a strong presence of $\mathrm{P}=\mathrm{O}$ bond for PS-PEI-Zr bonded to $20 \mu \mathrm{L}$ of demeton; while there is a considerable reduction of those bonds with $10 \mu \mathrm{L}$ of demeton and even disappearance for the actual compound not bonded to the nerve agent. Zirconium is an ideal candidate for immobilization of molecules with oxygen groups as contained in phosphates [44]. Because of zirconium's affinity for phosphoric groups, by treating the PEI modified colloids with zirconium we are able to amplify the interaction of the colloids with demeton's $\mathrm{P}=\mathrm{O}$ bond and thus see a significant change in the corresponding ATR spectrum. Thus, a similar sensor can be developed to sense other nerve agents with the organophosphate structure such as paraoxon. In contrast, if only PEI modified colloids were employed we would not expect to see a dramatic change in the ATR spectrum as there is no known specificity of $\mathrm{PEI}$ for the $\mathrm{P}=\mathrm{O}$ group of demeton. The ethyl group is distinguished at 1452 and $1492 \mathrm{~cm}^{-1}$ as a result of PS-PEI$\mathrm{Zr}$ bonding with demeton, explaining why it is absent in the curve of PS-PEI-Zr without demeton. These peaks strongly increase in intensity with increasing amounts of demeton solution. As the concentration of demeton decreased, it was observed that no strong peak of demeton is noticeable due to the weak signal, leading to much more prominent peaks when 10 and $20 \mu \mathrm{L}$ of demeton are present. Based on the response of the PS-PEI-Zr nanocomposites to the experimental parameters of the concentration of demeton in 1 liter of solution, the minimum sensitivity limit of this platform for the detection of demeton is approximately $2.0 \pm$ $0.2 \mathrm{PPB}$. This can be considered the effective dose which would result in a detectable change in the ATR spectrum. The above result allows us to propose a model for the development of chemical nerve agents through the use of ATR.

\section{Conclusion}

A simple method was developed to coat polystyrene colloids with zirconium particles. The zirconium nanoparticles were able to attach to polystyrene due to its modification with PEI, which increased the speed of the reaction and it had no appreciable effect on the formation of the core colloids. The detection of OPs has become important because many nerve agents and pesticides are organophosphates. The ability to detect small quantities and/or low concentrations of these compounds is critical. The strong bond formed between $\mathrm{Zr}$ and demeton allows for facile detection of the demeton peaks by using various characterization techniques. PS-PEI$\mathrm{Zr}$ is simple to synthesize and utilize, and its use to detect OPs is useful for applications such as pesticide analysis. The developed method is an efficient strategy due to its simple operation, easy large-scale production, and generality for various core colloids.

\section{Acknowledgments}

This work was supported by grants from the National Institutes of Health, RIMI program, Grant P20 MD001085, and the Department of Defense, Grant W911NF-06-1-0433 whose supports are greatly appreciated.

\section{References}

[1] F. Caruso, "Nanoengineering of particle surfaces," Advanced Materials, vol. 13, no. 1, pp. 11-22, 2001.

[2] S. J. Oldenburg, R. D. Averitt, S. L. Westcott, and N. J. Halas, "Nanoengineering of optical resonances," Chemical Physics Letters, vol. 288, no. 2-4, pp. 243-247, 1998.

[3] P. Schuetz and F. Caruso, "Semiconductor and metal nanoparticle formation on polymer spheres coated with weak polyelectrolyte multilayers," Chemistry of Materials, vol. 16, no. 16, pp. 3066-3073, 2004.

[4] A. Dawson and P. V. Kamat, "Semiconductor-metal nanocomposites. Photoinduced fusion and photocatalysis of goldcapped $\mathrm{TiO}_{2}\left(\mathrm{TiO}_{2}\right.$ /gold) nanoparticles," Journal of Physical Chemistry B, vol. 105, no. 5, pp. 960-966, 2001. 
[5] C. Pacholski, A. Kornowski, and H. Weller, "Site-specific photodeposition of silver on $\mathrm{ZnO}$ nanorods," Angewandte Chemie International Edition, vol. 43, no. 36, pp. 4774-4777, 2004.

[6] M. Jokob and H. Levanon, "Charge distribution between UVirradiated $\mathrm{TiO}_{2}$ and gold nanoparticles: determination of shift in the fermi level," Nano Letters, vol. 3, no. 3, p. 353, 2003.

[7] T. Ji, V. G. Lirtsman, Y. Avny, and D. Davidov, "Preparation, characterization, and application of Au-shell/polystyrene beads and Au-shell/magnetic beads," Advanced Materials, vol. 13, no. 16, pp. 1253-1256, 2001.

[8] B. Sadtler and A. Wei, "Spherical ensembles of gold nanoparticles on silica: electrostatic and size effects," Chemical Communications, no. 15, pp. 1604-1605, 2002.

[9] S.-E. Park, M.-Y. Park, P.-K. Han, and S.-W. Lee, "The effect of $\mathrm{pH}$-adjusted gold colloids on the formation of gold clusters over APTMS-coated silica cores," Bulletin of the Korean Chemical Society, vol. 27, no. 9, pp. 1341-1345, 2006.

[10] L. A. Belfiore and E. M. Indra, "Transition metal compatibilization of poly(vinylamine) and poly(ethylene imine)," Journal of Polymer Science Part B, vol. 38, no. 4, pp. 552-561, 2000.

[11] X. Sun, S. Dong, and E. Wang, "One-step synthesis and characterization of polyelectrolyte-protected gold nanoparticles through a thermal process," Polymer, vol. 45, no. 7, pp. 21812184, 2004.

[12] E. Prodan, C. Radloff, N. J. Halas, and P. Nordlander, "A hybridization model for the plasmon response of complex nanostructures," Science, vol. 302, no. 5644, pp. 419-422, 2003.

[13] S. A. Kalele, A. A. Kundu, S. W. Gosavi, et al., "Rapid detection of Escherichia coli by using antibody-conjugated silver nanoshells," Small, vol. 2, no. 3, pp. 335-338, 2006.

[14] S. W. Bishnoi, C. J. Rozell, C. S. Levin, et al., "All-optical nanoscale pH meter," Nano Letters, vol. 6, no. 8, pp. 16871692, 2006.

[15] F. Caruso, "Nanoengineering of particle surfaces," Advanced Materials, vol. 13, no. 1, pp. 11-22, 2001.

[16] U. Jeong, Y. Wang, M. Ibisate, and Y. Xia, "Some new developments in the synthesis, functionalization, and utilization of monodisperse colloidal spheres," Advanced Functional Materials, vol. 15, no. 12, pp. 1907-1921, 2005.

[17] V. Salgueiriño-Maceira, M. A. Correa-Duarte, M. Spasova, L. M. Liz-Marzán, and M. Farle, "Composite silica spheres with magnetic and luminescent functionalities," Advanced Functional Materials, vol. 16, no. 4, pp. 509-514, 2006.

[18] S. A. Kalele, S. S. Ashtaputre, N. Y. Hebalkar, et al., "Optical detection of antibody using silica-silver core-shell particles," Chemical Physics Letters, vol. 404, no. 1-3, pp. 136-141, 2005.

[19] J. P. Walker and S. A. Asher, "Acetylcholinesterase-based organophosphate nerve agent sensing photonic crystal," Analytical Chemistry, vol. 77, no. 6, pp. 1596-1600, 2005.

[20] T. L. Rosenberry, Advances in Enzymology and Related Areas of Molecular Biology, John Wiley \& Sons, New York, NY, USA, 1975.

[21] S. Zhang, H. Zhao, and R. John, "Development of a quantitative relationship between inhibition percentage and both incubation time and inhibitor concentration for inhibition biosensors-theoretical and practical considerations," Biosensors and Bioelectronics, vol. 16, no. 9-12, pp. 1119-1126, 2001.

[22] S. Fennouh, V. Casimiri, and C. Burstein, "Increased paraoxon detection with solvents using acetylcholinesterase inactivation measured with a choline oxidase biosensor," Biosensors and Bioelectronics, vol. 12, no. 2, pp. 97-104, 1997.
[23] J. Wang, "Microchip devices for detecting terrorist weapons," Analytica Chimica Acta, vol. 507, no. 1, pp. 3-10, 2004.

[24] O. A. Sadik, W. H. Land Jr., and J. Wang, "Targeting chemical and biological warfare agents at the molecular level," Electroanalysis, vol. 15, no. 14, pp. 1149-1159, 2003.

[25] Y. Lin, F. Lu, and J. Wang, "Disposable carbon nanotube modified screen-printed biosensor for amperometric detection of organophosphorus pesticides and nerve agents," Electroanalysis, vol. 16, no. 1-2, pp. 145-149, 2004.

[26] J. Sherma, "Pesticides," Analytical Chemistry, vol. 65, no. 12, pp. 40R-54R, 1993.

[27] B. Bucur, D. Fournier, A. Danet, and J.-L. Marty, "Biosensors based on highly sensitive acetylcholinesterases for enhanced carbamate insecticides detection," Analytica Chimica Acta, vol. 562, no. 1, pp. 115-121, 2006.

[28] J. C. Vidal, S. Esteban, J. Gil, and J. R. Castillo, "A comparative study of immobilization methods of a tyrosinase enzyme on electrodes and their application to the detection of dichlorvos organophosphorus insecticide," Talanta, vol. 68, no. 3, pp. 791-799, 2006.

[29] F. Mazzei, F. Botrè, S. Montilla, R. Pilloton, E. Podestà, and C. J. Botrè, "Alkaline phosphatase inhibition based electrochemical sensors for the detection of pesticides," Journal of Electroanalytical Chemistry, vol. 547, no. 1, pp. 95-100, 2004.

[30] C. Karnati, H. Du, H.-F. Ji, et al., "Organophosphorus hydrolase multilayer modified microcantilevers for organophosphorus detection," Biosensors and Bioelectronics, vol. 22, no. 11, pp. 2636-2642, 2007.

[31] P. Mulchandani, W. Chen, and A. Mulchandani, "Microbial biosensor for direct determination of nitrophenyl-substituted organophosphate nerve agents using genetically engineered Moraxella sp," Analytica Chimica Acta, vol. 568, no. 1-2, pp. 217-221, 2006.

[32] D. De Souza, S. A. S. Machado, and R. C. Pires, "Multiple square wave voltammetry for analytical determination of paraquat in natural water, food, and beverages using microelectrodes," Talanta, vol. 69, no. 5, pp. 1200-1207, 2006.

[33] V. A. Pedrosa, D. Miwa, S. A. S. Machado, and L. A. Avaca, "On the utilization of boron doped diamond electrode as a sensor for parathion and as an anode for electrochemical combustion of parathion," Electroanalysis, vol. 18, no. 16, pp. 1590-1597, 2007.

[34] H. El Bakouri, J. M. Palacios-Santander, L. Cubillana-Aguilera, A. Ouassini, I. Naranjo-Rodríguez, and J. L. H.-H. de Cisneros, "Electrochemical analysis of endosulfan using a C18-modified carbon-paste electrode," Chemosphere, vol. 60, no. 11, pp. 1565-1571, 2005.

[35] K. D. Dobson and A. J. McQuillan, "An infrared spectroscopic study of carbonate adsorption to zirconium dioxide sol-gel films from aqueous solutions," Langmuir, vol. 13, no. 13, pp. 3392-3396, 1997.

[36] Y. Lu, L. Han, C. J. Brinker, T. M. Niemczyk, and G. P. Lopez, "Chemical sensors based on hydrophobic porous sol-gel films and ATR-FTIR spectroscopy," Sensors and Actuators B, vol. 36, no. 1-3, pp. 517-521, 1996.

[37] B. J. Ninness, D. W. Bousfield, and C. P. Tripp, "In situ infrared technique for studying adsorption onto particulate silica surfaces from aqueous solutions," Applied Spectroscopy, vol. 55, no. 6, pp. 655-662, 2001.

[38] M. G. Baschetti, E. Piccinini, T. A. Barbari, and G. C. Sarti, "Quantitative analysis of polymer dilation during sorption using FTIR-ATR spectroscopy," Macromolecules, vol. 36, no. 25, pp. 9574-9584, 2003. 
[39] R. Göbel, R. W. Seitz, S. A. Tomellini, R. Krska, and R. Kellner, "Infrared attenuated total reflection spectroscopic investigations of the diffusion behaviour of chlorinated hydrocarbons into polymer membranes," Vibrational Spectroscopy, vol. 8, no. 2, pp. 141-149, 1995.

[40] U. Hellstern and V. Hoffmann, "Diffusion in ultrathin films studied by time resolved FTIR-ATR spectroscopy," Journal of Molecular Structure, vol. 349, pp. 329-332, 1995.

[41] C. Tian, E. Wang, L. Gao, et al., "A precursor route for the preparation of metal-dielectric composite in large scale," Chemistry Letters, vol. 35, no. 7, pp. 812-813, 2006.

[42] C. Tian, B. Mao, E. Wang, et al., "Simple strategy for preparation of core colloids modified with metal nanoparticles," The Journal of Physical Chemistry C, vol. 111, no. 9, pp. 3651-3657, 2007.

[43] B. Chaufer, M. Rabiller-Baudry, A. Bouguen, J. P. Labbé, and A. Quémerais, "Spectroscopic characterization of zirconia coated by polymers with amine groups," Langmuir, vol. 16, no. 4, pp. 1852-1860, 2000.

[44] M. Fang, D. M. Kaschak, A. C. Sutorik, and T. E. Mallouk, "A "mix and match" ionic-covalent strategy for self-assembly of inorganic multilayer films," Journal of the American Chemical Society, vol. 119, no. 50, pp. 12184-12191, 1997. 

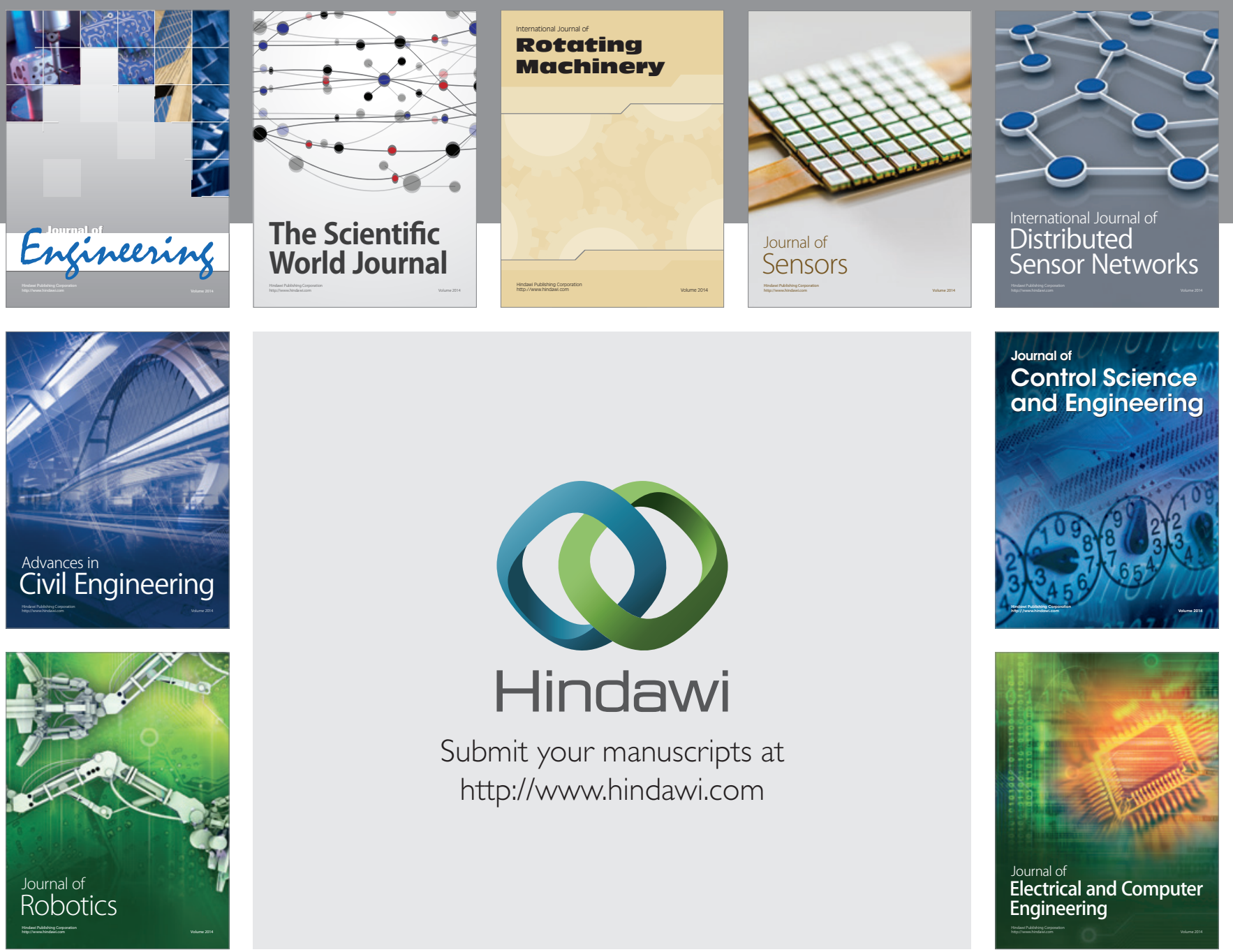

Submit your manuscripts at

http://www.hindawi.com
